\title{
A dynamic game formulation for control of opinion dynamics over social networks
}

\author{
Jomphop Veetaseveera, Vineeth S. Varma ${ }^{[0000-0001-8762-2790]}$, and \\ Irinel-Constantin Morărescu ${ }^{1}$ \\ Université de Lorraine, CRAN, UMR 7039 and the CNRS, CRAN, UMR 7039, \\ France, jomphop.veetaseveera@univ-lorraine.fr
}

\begin{abstract}
This paper considers the case where the opinion of agents in a social network is influenced not only by the other agents, but also by two marketers in competition. The main contributions of this work is to propose a dynamical game formulation of the problem and to conduct the corresponding equilibrium analysis. Due to the competition between the marketers, the opinions never reach consensus but are spread between the desired opinions of the two marketers. Our analysis provides practical insights to know how a marketer should exploit its knowledge about the social network to design the control of opinions using results from optimal control theory. Numerical examples illustrate the analysis.
\end{abstract}

\section{Introduction}

A duopoly is a standard problem in economics, politics, and marketing that considers the competition between two (dominant) players over a market, for example, see [10]. Illustrative examples of real-life duopolies are Airbus/Boeing in the market of large commercial airplanes, Republican/Democratic parties in the American politics.

Traditional research on competitive games between marketers assumes a homogeneous population of consumers $[7,6]$. Unlike these works, we propose a marketing resource allocation based on the influence power that each individual has over the (physical or digital) social network. Basically, we consider that the advertising is done in two steps: the first is done by the marketer that allocate her resources to sway some individuals/agents on her opinion and the second is done by the agents of the social network who influence each other. Consequently, each marketer has to target appropriate influential agents in the network in order to optimize her revenue. Since the focus of the paper is on the resource allocation of the marketer, the second step is modeled by a simple opinion dynamics model introduced in [4].

In this paper we consider the challenging problem that requires to minimize the distance between average of opinions and a desired value using a given control/marketing budget over a social network split in two groups. Basically, this social network with contrarian population represents a model for real cases such as supporters of competing teams, parties, etc. On top of this assumption on the 
network structure we also assume that the maximal marketing influence cannot instantaneously make the opinion of one individual to be equal with the desired value.

In the literature on viral marketing, the idea that members of a social network influence each other's purchasing decisions have been studied, with the goal being to select the best set of people to market to such that the overall profit is maximized by propagation of influence through the network [5]. This problem has since received much attention, including both empirical and theoretical results [1], but these results often consider a single entity influencing the network.

In this paper, we consider two competing marketers who want to use their marketing budget in order to sway on their side as many individuals of the network as possible. Thus, the natural framework to exploit is that of game theory and a reasonable solution concept (for arguments see e.g., [8]) for analyzing such a competition situation is the Nash equilibrium (NE). In [9], the authors consider multiple influential entities competing to control the opinion of consumers under a game theoretical setting. However, this work assumes an undirected graph and a voter model for opinion dynamics resulting in strategies that are independent of the node centrality (i.e., agent influence power). On the other hand, the recently published work [11] considers a similar competition with opinion dynamics over a directed graph but with no budget constraints and by considering the average agents' opinion instead of the final one; these two differences change the problem significantly.

Notation. Let $\mathbb{R}:=(-\infty, \infty)$ and $\mathbb{R}_{\geq 0}:=[0, \infty)$. We use $I_{n}$ for the identity matrix, $\mathbf{1}_{n}$ for the column vector of 1 and $\mathbf{0}_{n}$ for the column vector of $0, n \in \mathbb{N}$. In the sequel, the symbol $\|$.$\| corresponds to the norm 2$ and $x^{\top}$ stands for the transpose of $x, x \in \mathbb{R}^{n}$.

\section{Problem statement}

We consider a social network populated by agents belonging to the set $\mathcal{V}:=$ $\{1,2, \ldots, n\}$. The parameters $a_{i, j}$ characterize the influence of agent $j$ on agent $i, i \neq j$ and $i, j \in \mathcal{V}$. The opinions of agent $i$ at time step $k$ is denoted by $x_{i}(k)$ and evolves according to

$$
x_{i}(k+1)=x_{i}(k)+\delta\left(\sum_{j \in \mathcal{V}} a_{i, j}\left(x_{j}(k)-x_{i}(k)\right)\right),
$$

due to their interaction with other agents in the social network. In our problem, we consider the case where these agents are also influenced by the marketing/advertising of two marketers. The desired opinion of Marketer $m$ is $d_{m} \in \mathbb{R}$, $m \in\{1,2\}$. As a result of these interactions, the opinion dynamics for $x(k)$, the collective vector of all opinions is given by

$$
x(k+1)=D x(k)+B_{1} u_{1}(k)+B_{2} u_{2}(k),
$$


where $D=I_{n}-\delta L$ is the row stochastic matrix defining the internal dynamics of the network with $L \in \mathbb{R}^{n \times n}$ being the Laplacian matrix associated with adjacency $a_{i, j}, B_{m}$ denotes the manner in which the Marketers influence the agents and $u_{m}$ is the action of Marketers $m$ on the agents. We concentrate on two influence models:

1. Uniform broadcasting $(U B)$ with $B_{m}=(1,1, \ldots, 1)^{\top}$ which implies that all agents in the network receive the same control. This influence model corresponds to traditional advertising/marketing done on television or radio where the control is applied uniformly on all agents.

2. Targeted advertising $(T A)$ with $B_{m}=I_{n}$ which implies that the advertising control can be designed for each individual in the network. This model corresponds to modern social media marketing as done by companies like Facebook or Google.

Let $\mathbf{u}_{m}$ denote the sequence of control actions applied by Marketer $m$. We define the infinite horizon cost for Marketer $m$ as

$$
J_{m}\left(\mathbf{u}_{1}, \mathbf{u}_{2}\right):=\sum_{k=0}^{\infty} \alpha^{k}\left(\left\|x(k)-d_{m} \mathbf{1}_{n}\right\|^{2}+u_{m}(k)^{T} R_{m} u_{m}(k)\right),
$$

where $\alpha \in(0,1)$ is a discount factor which is often related to inflation rates/interest rates in economic literature. We assume that the revenue generated by the firm associated to Marketer $m$ at time $k$ depends on the market share captured by the firm. As the distance between the opinions of agents in the social network and the desired opinion $d_{m}$ decreases, the revenue increases. Alternately, we say that the loss incurred by not capturing the entire market is characterized by $\sum_{i \in \mathcal{V}}\left\|x_{i}(k)-d_{m}\right\|^{2}$. On the other hand, advertising to agent $i$ incurs a cost which we take to be $u_{m}^{T} R_{m} u_{m}$. The term $R_{m}$ is like the price for advertisements.

\section{Analysis}

Unlike standard control theory problems, in our framework, we have two competing marketers who attempt to bring $x_{i}(k)$ to a desired opinion $d_{m} \in \mathbb{R}$ with $d_{1} \neq d_{2}$. If $d_{1}=d_{2}$, the problem can be seen as a distributed optimal control problem. However, when $d_{1} \neq d_{2}$, each marketer has its own objective resulting in a non-cooperative game. Due to the cost function depending on $x(k)$ and its dynamics, we have a dynamic game [2].

The problem we consider corresponds to a difference-game as introduced in [2]. However, since these games are hard to analyse in the most general case, we make the following assumption.

Assumption 1 We assume that each marketer applies a static state feedback strategy,

$$
u_{m}(k)=G_{m}\left(d_{m} \mathbf{1}_{n}-x(k)\right)-M_{m},
$$

where $G_{m} \in \mathbb{R}^{n \times n}$ is a fixed feedback gain and $M_{m} \in \mathbb{R}^{n}$ is an offset applied to balance the opposing marketer resulting in the strategy given by $K_{m}:=$ $\left(G_{1} M_{1}\right) \in \mathbb{R}^{n \times n+1}$. 
We can redefine the cost $J_{m}$ in terms of the static feedback strategy $K_{m}$ as

$$
J_{m}\left(K_{1}, K_{2}\right):=\sum_{k=0}^{+\infty} \alpha^{k}\left(\left\|x(k)-d_{m} \mathbf{1}_{n}\right\|^{2}+u_{m}(k)^{T} R_{m} u_{m}(k)\right),
$$

where $x(k)$ follows $(2)$ and $u_{m}(k)$ is given by (4).

Under Assumption 1, we can reformulate the dynamic game with actions $\mathbf{u}_{m}$ as a static one-shot game with the action space of each player corresponding to being the control gain and offset. Formally, we define the game $\mathcal{G}$ in strategic form as follows

$$
\mathcal{G}:=\left(\{1,2\},\left\{\mathcal{K}_{1}, \mathcal{K}_{2}\right\},\left\{J_{1}, J_{2}\right\}\right)
$$

where:

1. $\{1,2\}$ is the set of players or Marketers;

2. $\mathcal{K}_{m}$ is the set of pure actions for player $m$, specifically, we have $K_{m}=$ $\left(G_{1} M_{1}\right) \in \mathcal{K}_{m}=\mathbb{R}^{n \times(n+1)} ;$

3. $J_{m}\left(K_{1}, K_{2}\right)$ as defined in (5) is the cost function for player $m$;

A natural solution concept for a game is that of the Nash equilibrium. For convenience, we use $-m$ to denote the other player, i.e. $3-m$ for all $m \in\{1,2\}$. A pure Nash equilibrium (NE) is defined for the game $\mathcal{G}$ as follows.

Definition 1. We say that $\left(K_{1}^{*}, K_{2}^{*}\right)$ form a $N E$ of the game $\mathcal{G}$ if and only if

$$
J_{m}\left(K_{m}, K_{-m}^{*}\right) \geq J_{m}\left(K_{m}^{*}, K_{-m}^{*}\right),
$$

for all $K_{m} \in \mathcal{K}_{m}$ and for all $m \in\{1,2\}$.

The NE is a suitable notion to study player interactions in a non-cooperative game when all players behave rationally and are capable of computing the best decisions to make for a given opponent strategy. However, this assumption on player behavior may not always hold and some players may behave differently or play simpler/naive strategies.

As a first step, we consider the case where player 2 is naive, i.e. it plays a given strategy $K_{2}$. In the following, we compute the best response player $m$ can do for a given strategy player $K_{-m}$ played by $-m$.

\subsection{Best response to a given opponent strategy}

Let $\beta_{m}\left(K_{-m}\right)$ denote the best response function defined as

$$
\beta_{m}\left(K_{-m}\right)=\arg \min _{K_{m}} J_{m}\left(K_{m}, K_{-m}\right) .
$$

In general, the best response function is set valued as the $\arg \min$ is not unique. However, the following proposition provides a unique best response and the method to find this value. 
Proposition 1. The best response function is unique and can be evaluated as

$$
\beta_{m}\left(K_{-m}\right)=\alpha\left(\tilde{B}_{m}^{\top} P_{m} \tilde{B}_{m}+R_{m}\right)^{-1} \tilde{B}_{m}^{\top} P_{m} \tilde{D}_{m},
$$

where $\tilde{D}_{m}=\left(\begin{array}{cc}D-B_{-m} G_{-m} & B_{-m}\left(G_{-m} \mathbf{1}_{n}\left(d_{-m}-d_{m}\right)-M_{-m}\right) \\ \mathbf{0}_{n}^{\top} & 1\end{array}\right), \tilde{B}_{m}=\left(\begin{array}{c}B_{m} \\ \mathbf{0}_{n}^{\top}\end{array}\right)$ and $P_{m}$ is the solution to the Algebraic Riccati equation

$$
P_{m}=\tilde{D}_{m}^{\top}\left(\alpha P_{m}-\alpha^{2} P_{m} \tilde{B}_{m}\left(\alpha \tilde{B}_{m}^{\top} P_{m} \tilde{B}_{m}+R_{m}\right)^{-1} \tilde{B}_{m}^{\top} P_{m}\right) \tilde{D}_{m}+Q_{m},
$$

where $Q_{m}=\left(\begin{array}{cc}I_{n} & \mathbf{0}_{n} \cdot \\ \mathbf{0}_{n}^{\top} & 1\end{array}\right)$

Proof. Here, we search for $u_{1}^{*}$ minimizing the cost $J_{1}\left(u_{1}, u_{2}\right)$ supposing $u_{2}$ is known. The reasoning is still the same to find $u_{2}^{*}$ for $u_{1}$ known. Under Assumption 1 , we have

$$
x(k+1)=D x(k)+B_{1} u_{1}(k)+B_{2} G_{2}\left(d_{2} \mathbf{1}_{n}-x(k)\right)-B_{2} M_{2} .
$$

Let $e_{1}(k)=x(k)-d_{1} \mathbf{1}_{n}$ be the error for the desired opinion $d_{1}$. The error dynamics is

$$
e_{1}(k+1)=\left(D-B_{2} G_{2}\right) e_{1}(k)+B_{1} u_{1}(k)+B_{2} G_{2} \mathbf{1}_{n}\left(d_{2}-d_{1}\right)-B_{2} M_{2},
$$

where $B_{2} G_{2} \mathbf{1}_{n}\left(d_{2}-d_{1}\right)-B_{2} M_{2}$ is a constant affine term. We modify (12) by including the affine term in the state variable and we use an algorithm from the Section 4.2 of Vol. II, 4th Ed. of [3], to solve (10). The modified system is

$$
\left(\begin{array}{c}
e_{1}(k+1) \\
1
\end{array}\right)=\tilde{D}_{1}\left(\begin{array}{c}
e_{1}(k) \\
1
\end{array}\right)+\tilde{B}_{1} u_{1}
$$

where $\tilde{D}_{1}=\left(\begin{array}{cc}D-B_{2} G_{2} & B_{2} G_{2} \mathbf{1}_{n}\left(d_{2}-d_{1}\right)-B_{2} M_{2} \\ \mathbf{0}_{n}^{\top} & 1\end{array}\right)$ and $\tilde{B}_{1}=\left(\begin{array}{c}B_{1} \\ \mathbf{0}_{n}^{\top}\end{array}\right)$.

Finally, the optimal control $u_{1}^{*}$ depending on $u_{2}$ is

$$
\begin{aligned}
u_{1}^{*}(x) & =-\alpha\left(\alpha \tilde{B}_{1}^{\top} P_{1} \tilde{B}_{1}+R_{1}\right)^{-1} \tilde{B}_{1}^{\top} P_{1} \tilde{D}_{1}\left(\begin{array}{c}
e_{1} \\
1
\end{array}\right) \\
& =-\left(G_{1} M_{1}\right)\left(\begin{array}{c}
e_{1} \\
1
\end{array}\right)=-G_{1}\left(x-d_{1}\right)-M_{1},
\end{aligned}
$$

which is consistent with Assumption 1. Thus, the the best response is $\beta_{m}\left(K_{-m}\right)=$ $\left(G_{1} M_{1}\right)$. The uniqueness of the solution comes from the uniqueness of the Riccati solution.

\subsection{Nash equilibrium}

We propose the following iterative asynchronous best response algorithm to find a Nash equilibrium of the game $\mathcal{G}$. While the convergence of the iterative best response dynamics, i.e., Algorithm 1 is not guaranteed for all game classes, our numerical tests show that this property holds as illustrated in the next section. 


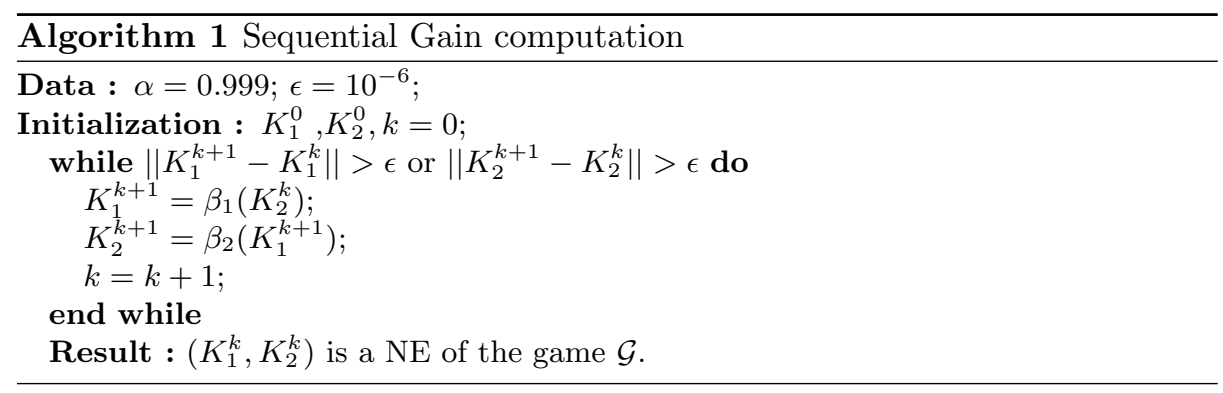

\section{Numerical illustration}

In this section, we will study the performance of targeted advertising $(T A)$ when compared to uniform broadcasting $(U B)$. For all our numerical tests, we will consider the graph presented in Figure 1 . We take $d_{1}=2$ and $d_{2}=-2$. When player $m$ is implementing $T A$, we take $R_{m} \in\left\{I_{n}, 2 I_{n}\right\}$, and $R_{m} \in\{n, 2 n\}$ while implementing $U B$. We consider the following initial conditions

$$
x_{A}=(1,2,-3,0,6,-5,4,3,-2,4)^{\top} \text { and } x_{B}=(4,-2,-2,-3,2,0,2,-1,1,0)^{\top} .
$$

As a first step, we consider the situation when both marketers apply the NE strategy, but when Marketer 1 applies TA and Marketer $2 U B$. Since for $U B$, the control $u_{2}$ is applied to all $n$ agents, we take $R_{2}=n$ and $R_{1}=I_{n}$ to look at a symmetric situation in terms of the Marketer revenue and cost. If we take a graph where all agents have the same centrality, we notice that the opinions of all agents converge to 0 which lies at the middle of $d_{1}$ and $d_{2}$ due to the symmetry.

However, if we consider the graph as in Figure 1 with $x(0)=x_{A}$, the opinions evolve as seen in Figure 2 with the average final opinion being closer to $d_{1}$, the desired opinion of Marketer 1. This demonstrates the advantage of $T A$ and Table 1 further illustrates how Marketer 1 prioritizes advertising the agents with a higher centrality (vector centrality associated to the Laplacian matrix).

\begin{tabular}{|c|c|c|c|c|c|c|c|c|c|c|}
\hline agent & 1 & 2 & 3 & 4 & 5 & 6 & 7 & 8 & 9 & 10 \\
\hline Centrality & 0.476 & 0.068 & 0.006 & 0.011 & 0.231 & 0.003 & 0.003 & 0.006 & 0.162 & 0.034 \\
\hline$x_{i}(t \mapsto \infty)$ & 2.484 & 0.959 & 0.550 & 0.418 & 1.692 & 0.024 & -0.864 & 0.206 & 1.188 & -0.144 \\
\hline \multicolumn{8}{c|}{ Table 1: Centrality of each agent }
\end{tabular}

In Figure 3 and Figure 4, we plot the evolution of average opinion when both Marketers apply $T A$ or $U B$ respectively. Interestingly, consensus is reached when TA is applied only when $R_{1}=R_{2}$ and in this case all the opinions converge to 0 which lies halfway between $d_{1}$ and $d_{2}$. However, taking $R_{2}=2 R_{2}$ results in the opinions converging to

$$
\lim _{t \rightarrow \infty} x(t)=(2.48 ; 0.96 ; 0.55 ; 0.42 ; 1.69 ; 0.02 ;-0.86 ; 0.2055 ; 1.19 ;-0.14)^{T} .
$$




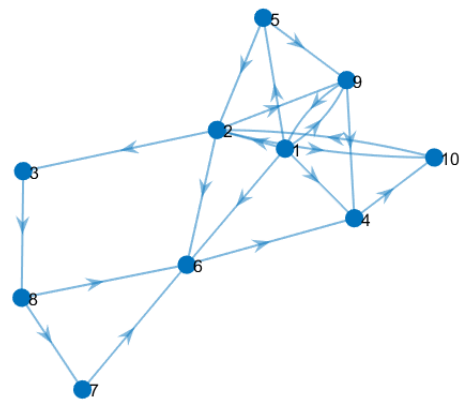

Fig. 1: Directed graph of 10 agents.

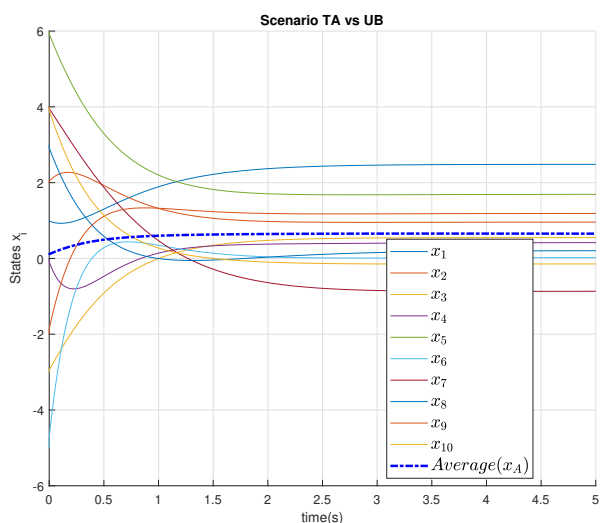

Fig. 2: Opinion dynamics with $R_{1}=I_{n}$ and $R_{2}=n$.

We notice that for all $R_{m}, U B$ vs $U B$ results in consensus. The corresponding costs are provided in Table 2 .
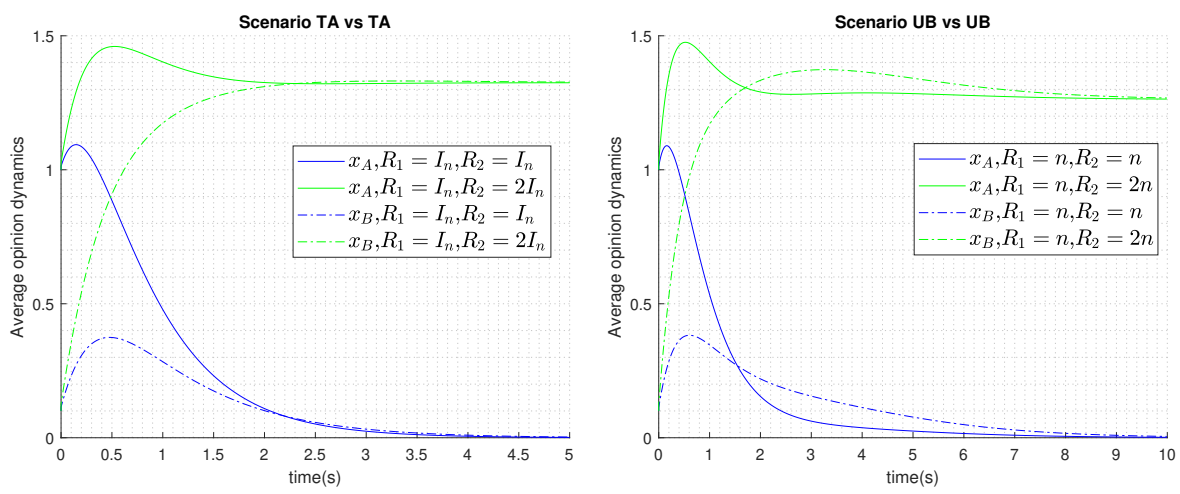

Fig. 3: Opinion dynamics in the scenario Fig. 4: Opinion dynamics in the scenario $T A$

$U B$

\section{Conclusion}

In this work, we have studied the behavior of two competing firms/marketers that control the opinions of a set of consumers interacting over a social network. We consider a linear interaction model and quadratic costs for each firm related 


\begin{tabular}{|c|c|c|c|c|c|c|}
\hline \multirow{2}{*}{ Scenario } & \multirow{2}{*}{$R_{1}$} & \multirow{2}{*}{$R_{2}$} & \multicolumn{4}{|c|}{ Cost with $K^{*}$} \\
\hline & & & $J_{1}\left(x_{A}\right)$ & $J_{2}\left(x_{A}\right)$ & $J_{1}\left(x_{B}\right)$ & $J_{2}\left(x_{B}\right)$ \\
\hline \multirow{2}{*}{$T A$} & $I_{n}$ & $I_{n}$ & 86453 & 102759 & 87233 & 98272 \\
\hline & $\overline{I_{n}}$ & $2 I_{n}$ & 30731 & 127644 & 31428 & 122783 \\
\hline \multirow{2}{*}{$U B$} & $n$ & $n$ & 77755 & 97619 & 78200 & 97093 \\
\hline & $n$ & $2 n$ & 29549 & 122263 & 30163 & 120843 \\
\hline$T A$ vs $U B$ & $I_{n}$ & $n$ & 54367 & 120470 & 56075 & 115919 \\
\hline
\end{tabular}

Table 2: Costs depending on the scenario and the initial condition

to the revenue earned and the amount they spent in order to control the network (via advertisements or other marketing strategies). As a first step, we find the optimal static state feedback control which must be applied by a marketer assuming that the other marketer applies a given strategy. Next, we provide an iterative algorithm, which we observe to converge to the Nash equilibrium after extensive numerical tests. We provide numerical examples which illustrate the advantage of viral marketing techniques (targeted advertising based on the social graph) when compared to traditional advertising strategies (uniform broadcast). Future work will focus on theoretically studying the existence and uniqueness of the Nash equilibrium of the game we have studied.

\section{References}

1. Arthur, D., Motwani, R., Sharma, A., Xu, Y.: Pricing strategies for viral marketing on social networks. In: International Workshop on Internet and Network Economics. pp. 101-112. Springer (2009)

2. Basar, T., Olsder, G.J.: Dynamic noncooperative game theory, vol. 23. Siam (1999)

3. Bertsekas, D.P., Bertsekas, D.P., Bertsekas, D.P., Bertsekas, D.P.: Dynamic programming and optimal control, vol. 1. Athena scientific Belmont, MA (1995)

4. DeGroot, M.H.: Reaching a consensus. Journal of the American Statistical Association 69(345), 118-121 (1974)

5. Domingos, P., Richardson, M.: Mining the network value of customers. In: Proceedings of the seventh ACM SIGKDD international conference on Knowledge discovery and data mining. pp. 57-66. ACM (2001)

6. Esmaeili, M., Aryanezhad, M.B., Zeephongsekul, P.: A game theory approach in seller-buyer supply chain. European Journal of Operational Research 195(2), 442448 (2009)

7. Friedman, L.: Game-theory models in the allocation of advertising expenditures. Operations Research 6(5), 699-709 (1958)

8. Lasaulce, S., Tembine, H.: Game Theory and Learning for Wireless Networks : Fundamentals and Applications. Academic Press (2011)

9. Masucci, A.M., Silva, A.: Strategic resource allocation for competitive influence in social networks. In: Communication, Control, and Computing (Allerton), 2014 52nd Annual Allerton Conference on. pp. 951-958. IEEE (2014)

10. Singh, N., Vives, X.: Price and quantity competition in a differentiated duopoly. The RAND Journal of Economics pp. 546-554 (1984)

11. Varma, V., Morarescu, I.C., Lasaulce, S., Martin, S.: Opinion dynamics aware marketing strategies in duopolies. In: 56th IEEE Conference on Decision and Control, CDC 2017 (2017) 